\title{
552 CASOS DE ECLAMPSIA TRATADOS EN EL INSTITUTO MATERNO INFANTIL DE BOGOTA*
}

\author{
Dr. Roberto Páez Vargas \\ Del Instituto Materno Infantil de Bogotá.
}

\section{INTRODUCCION}

Desde la época más remota, cuando la inyección retromamaria de yoduro de potasio y aire, la mastectomía radical, la decapsulación renal, la trepanación del cráneo, la venisección masiva y la punción lumbar con extracción de gran cantidad de líquido cefalorraquídeo eran los procedimientos usuales en el tratamiento de la Eclampsia, hasta nuestros días cuando una gran cantidad de fármacos ha enriquecido la terapéutica, han sido numerosos los tratamientos propuestos para el control de la gestosis en su etapa convulsiva. Pese a ese caudal de medicamentos, en otros medios científicos, el tratamiento de la toxemia aguda no ha disminuído los porcentajes estadísticos de morbi-mortalidad por tan grave complicación.

La mayor parte de los planes terapéuticos tienen como fundamento fisiopatológico un terreno hipotético y por consiguiente se resienten de empirismo. Casi todos tienden a controlar la toxemia desde un punto de vista sintomático sin adentrarse en las

\footnotetext{
* Trabajo presentado para ingreso a la Sociedad Colombiana de Obstetricia y Ginecolo-
} gía. razones etiopatogénicas del cuadro clínico. Los vacíos en cuanto a la esencia o causa fundamental de la toxemia explican en parte este planteamiento. Sinembargo, las modernas adquisiciones que ha aportado la endocrinología y la medicina psicosomática, han permitido la resolución de algunos de los interrogantes planteados desde tiempo inmemorial dentro de la averiguación etiológica de la preeclampsia-eclampsia.

Las nuevas técnicas de investigación nefrológica: Bacteriológicas, radiológicas e histopatológicas, han permitido deslindar cuadros nosológicos clasificados otrora dentro de los padecimientos propios de la gestación, para concretar así los verdaderos aspectos orgánicos de la toxemia del embarazo.

A la par con la investigación puramente clínica ha marchado la farmacología en tal forma que hoy se dispone de una serie de medicamentos que han ampliado el campo de acción terapéutico, modificándose, como se verá más adelante, de una manera radical y promisoria el oscuro pronóstico del más grave padecimiento inherente a la gestación. 
Con fines ilustrativos hemos de recordar, de acuerdo con Dieckman, citado por Walker (1) los períodos y procedimientos seguidos a través de muchos años en el tratamiento de la Eclampsia.

Primer Período: Comienza en 1745 y dura aproximadamente 100 años. Se caracterizó por la administración de purgantes drásticos, sangría y sedantes.

Período Segundo: Se inicia en 1845 y dura 50 años: Utilización del veratrum viride y apresuramiento del parto mediante dilatación manual del cuello.

Tercer y Cuarto Períodos: Son sucesivos y se consideran ultrarradicales, se extienden desde 1895 hasta 1915; cesárea abdomina!, histerotomía vaginal y parto forzado, caracterizan esta etapa.

Quinto Período: Se extiende desde 1915 hasta 1927; la administración de glucosa hipertónica, la sedación, la estimulación de la eliminación y la introducción por Titus en 1920 del sulfato de magnesio por vía parenteral en el tratamiento de la toxemia, individualizan este período.

El último período se inicia en 1928 y se extiende hasta la actualidad. Se caracteriza por una mejor atención obstétrica y el aprovechamiento de los procedimientos de los períodos anteriores con prescindencia de los medios que pudieran resultar peligrosos para la supervivencia fetal.

Es nuestra intención fundamental presentar un análisis estadístico de los diversos tipos de tratamiento empleados en el curso de los últimos años en el INSTITUTO MATERNO INFANTIL DE BOGOTA.

La inusitada frecuencia de la toxemia de la gestación en nuestro me- dio, impuso la creación de un Servicio dedicado a su control y tratamiento, dentro de la Institución mencionada. De sus archivos hemos obtenido gran parte del material recopilado en el presente estudio. Los resultados estadísticos que expondremos adelante permitirán hacer un análisis y mostrarán la evolución a través de los últimos tiempos. Nos abstendremos de entrar en detalles clínicos, fisiopatológicos y evolutivos que han sido tratados de manera extensa en comunicaciones y trabajos de personal de dicho servicio (2) (3), con anterioridad.

Nuestro análisis comparativo se establece entre 4 grupos de tratamientos empleados a partir de 1954. Los resultados obtenidos antes de dicho año fueron dados a conocer en su oportunidad (4) y nos servirán para parangonar las cifras al final de este trabajo.

Veremos en detalle los distintos grupos y tipos de tratamiento empleados.

\section{PRIMER GRUPO}

Se utilizó durante los años 1954 y parte de 1955. Comprende tres tipos de Tratamiento empleados de acuerdo con la gravedad del caso o por fracaso del anterior.

Tratamiento № 1. A base de: Dextrosa más Clorpromazina vía I.V. y Sulfato de Magnesio I.M. Ocasionalmente se adicionaba de Dolosal o Morfina de acuerdo con la severidad del proceso.

Tratamiento $\mathbf{N}: 2$. Se suministraba por fracaso del Ni 1 así: Dextrosa más Pentotal por vía intravenosa.

Tratamiento $\mathbf{N}$ : 3. Se limitaba a la intervención cesárea. A ella se recurría casi de manera sistemática aún antes de ensayar los tratamientos 1 y 2. En otras ocasiones se apelaba a 
ella por fracaso con los tratamientos anteriores. Al analizar los distintos tipos de parto veremos la enorme incidencia de la cesárea como procedimiento terapéutico de la Eclampsia y sin que para su ejecución mediara factor obstétrico diferente.

\section{SEGUNDO GRUPO}

Se utilizó desde mediados de 1955 hasta el final del año 1956. Consistía en la inyección intrarraquídea de sulfato de magnesio.

\section{TERCER GRUPO}

Fué puesto en práctica durante el año de 1958 y los primeros meses de 1959. Comprendía los siguientes tipos:

Tratamiento № 1. Dextrosa al 10 - $33 \%$ más 50 a 100 mlgrs. de Clorpromazina, vía I.V.

Tratamiento № 2. Indicado en la Eclampsia del preparto o del parto. Consistía en la Inyección intrarraquídea de 3 c.c. de Sulfato de Magnesio al 20\% más 50 mlgrs. de Clorpromazina en 500 c.c. de Dextrosa al $10 \%$.

Tratamiento $\mathrm{N}$ : 3. Indicado en la Eclampsia del preparto 0 del parto con feto muerto in útero así como también en la Eclampsia del postparto: $1 / 2$ a $1 \mathrm{gr}$. de Pentotal sódico en 500 a 1.000 c.c. de Dextrosa al $10 \%$ más inhalación simultánea de oxígeno.

Estos tratamientos se empleaban en la mayoría de los casos de manera combinada o cuando se fracasaba con uno de ellos. Así: Primero administración del № 1 y luego el № 2. Se pasaba al $\mathrm{N}$ : 3 por fallecimiento del feto.

\section{CUARTO GRUPO}

Es el Tratamiento "I.M.I." utilizado en la actualidad. Se puso en prác- tica desde Marzo de 1959. Su sistematización quedó planteada en una publicación de la Revista Colombiana de Obstetricia y Ginecología (3) y reafirmada en el Tercer Congreso Uruguayo de Ginecotocología en el año 1960. Consiste en la aplicación inicial y simultánea de: 200 miligramos de Fenobarbital por vía venosa y 200 mlgrs. por vía intramuscular, 4 a 6 miligramos de Acepromazina por vía intramuscular y 15 c.c. de Sulfato de Magnesia al $20 \%$ por vía venosa. Posteriormente se administra Clorotiazida por vía oral. La medicación se repite y combina de acuerdo con la evolución clínica y las necesidades de cada paciente. Los cuidados generales, el suministro de Oxígeno y la buena conducción obstétrica son fundamentales. Las bases fisiopatológicas del método fueron ampliamente expuestas (3).

\section{MATERIAL Y METODOS}

Para la elaboración de este trabajo consultamos los Archivos del Instituto Materno Infantil y del Servicio de Toxemias del mismo Instituto. Hallamos un total de 552 casos de Eclampsia de Enero de 1954 a 31 de Diciembre de 1962 correspondiendo a cada uno de los grupos de tratamiento el siguiente número de casos y haciendo la salvedad de que durante el año 1957 no se contabilizaron casos por fallas en los Archivos.

$\begin{array}{lrc}\text { Grupo N: } 1 & 98 \text { casos } \\ \text { Grupo N: } 2 & 41 & \text { " } \\ \text { Grupo N: } 3 & 67 & \text { " } \\ \text { Grupo N: } 4 & 346 ~ " \\ \text { TOTAL } & 552 \text { casos }\end{array}$

En las páginas siguientes veremos los resultados con cada uno de los Grupos de tratamientos para hacer luego el estudio comparativo. 
PRIMER GRUPO

\begin{tabular}{|c|c|c|c|c|c|}
\hline TIPO DE ECLAMPSIA & Anteparto & Intraparto & Postparto & & Mort./Global \\
\hline Total de Casos & 38 & 56 & 4 & & \\
\hline Feto vivo al ingreso & 33 & 51 & & & \\
\hline Feto muerto al ingreso & 0 & 5 & & & \\
\hline Abortos & 5 & - & & & \\
\hline Repitieron convuls. desp. trat. & 19 & 24 & 0 & & \\
\hline NO repitieron convuls. desp. trat. & 19 & 32 & & & \\
\hline PARIDAD Primíparas & 23 & 44 & 2 & & \\
\hline Secundíparas & 6 & 5 & & & \\
\hline Tercíparas y más & 9 & 7 & 2 & & \\
\hline PARTOS Espontáneos & 18 & 16 & & & \\
\hline Inducidos & 1 & & & & \\
\hline Fórceps & 3 & 14 & & & \\
\hline Cesáreas & 9 & 22 & & & \\
\hline Ventosas & 1 & 0 & & & \\
\hline Sin parto & 6 & 0 & & & \\
\hline Peso Fetal 1.000 a 1.500 grs. & 3 & 0 & & & \\
\hline 1.501 a 2.000 & 8 & 8 & & & \\
\hline 2.001 a 2.500 & 9 & 14 & & & \\
\hline 2.501 a 3.000 & 7 & 20 & & & \\
\hline 3.001 a 3.500 & 4 & 6 & & & \\
\hline 3.501 a 4.000 & 0 & 3 & & & \\
\hline Sin dato & 7 & 0 & & & \\
\hline Feto al nacer VIVOS & 27 & 45 & & & \\
\hline MUERTOS & 6 & 11 & & 17 & $=18.08 \%$ \\
\hline Mortalidad Perinatal & 8 & 7 & & 15 & $=16.80 \%$ \\
\hline Mortalidad Fetal Global & 14 & 18 & & 32 & $=34.04 \%$ \\
\hline Mortalidad Materna & 7 & 10 & 1 & 18 & $=18.36 \%$ \\
\hline
\end{tabular}

\section{PRIMER GRUPO}

Como se observa, La Mortalidad Materna para este primer grupo es de $18.36 \%$ y las causas se detallan en los siguientes resúmenes de las historias clínicas:

\section{ECLAMPSIA ANTEPARTO: 7}

1) Historia № $\mathbf{3 . 8 8 8}$, paciente primigestante que muere a las 48 horas de su ingreso, presenta ataques subintrantes que no ceden al tratamiento, entra en coma y muere 15 horas después sin haberse efectuado el parto. Embarazo de 32 semanas.

2 ) Historia No 4.288, paciente secundigestante que fallece a las 8 y media horas de su ingreso. Presenta numerosos ataques que no ceden al tratamiento. Coma y muerte.

3) Historia № 4.470, paciente secundigestante que ingresó en preeclampsia, hizo un ataque después del tratamiento. Coma y muerte a las
5 y media horas de su ingreso. Embarazo de 28 semanas.

4) Historia $\mathbf{N}^{\mathbf{0}} \mathbf{8 . 5 7 7}$, paciente multigestante que muere a las 42 horas de su ingreso. Numerosos ataques. Coma y muerte.

5) Historia No $\mathbf{8 . 3 9 9 ,}$, paciente secundigestante con embarazo de 22 semanas que hace tres ataques antes del tratamiento y 10 después. Coma y muerte. Permaneció en el Instituto 10 días.

6) Historia $\mathrm{N}^{0}$ 10.297, paciente multigestatnte, que presenta 5 ataques antes del tratamiento y 1 después; coma y defunción a las 14 horas de su ingreso. Embarazo de 28 semanas.

7) Historia $\mathbf{N}^{\mathbf{0}} \mathbf{1 1 . 7 7 2}$, paciente que presenta 3 ataques antes del tratamiento y 2 después. Entra en coma y muere a las cinco horas veinte minutos de su ingreso. Embarazo de 28 semanas. 


\section{ECLAMPSIA INTRAPARTO: 10}

1) Historia $\mathbf{N}^{0} \mathbf{4 . 1 8 2}$, paciente que ingresó con signos neurológicos de hemorragia cerebral. Defunción. Cesárea Post mortem.

2) Historia $\mathbf{N}^{0}$ 6.006, paciente que presentó numerosos ataques antes del ingreso, presentó signos de hemorragia cerebrat y fallece a las 12 horas después del ingreso.

3) Historia $\mathbf{N}^{0}$ 14.816, paciente que presentó numerosos ataques y cuyo número no se pudo precisar, entra en coma y fallece. No autopsia.

4) Historia $N^{0}$ 17.745, paciente que hizo 3 ataques antes del tratamiento. Coma y defunción.

5) Historia $\mathrm{N}^{0}$ 19.996, paciente que presentó 7 ataques antes del tratamiento y 1 después. Coma y muerte a las 20 horas de su ingreso.

6) Historia $\mathrm{N}^{0}$ 19.322, paciente que ingresó en coma y falleció a las 20 horas de su ingreso.
7) Historia $\mathrm{N}^{0}$ 20.711, paciente que presentó 1 ataque antes del tratamiento y 6 después. Coma y defunción.

8) Historia $\mathbf{N}^{0}$ 20.588, paciente que presentó 8 ataques antes de la cesárea raquianestesia. Apnea y muerte. Cesárea Post mortem.

9) Historia $\mathbf{N}^{0}$ 23.465, paciente que presentó 6 ataques antes del tratamiento y 2 después. Parto espontáneamente y muerte 3 horas después.

10) Historia $\mathbf{N}^{o} \mathbf{2 9 . 5 8 8}$, paciente que ingresó en coma, presentó numerosos ataques antes del tratamiento. Muere a las pocas horas de hospitalizada.

\section{ECLAMPSIA POSTPARTO: 1}

1) Historai $N^{0}$ 6.028, paciente que tiene parto gemelar, presenta un ataque $y$ muere.

\section{SEGUNDO GRUPO}

\begin{tabular}{|c|c|c|c|c|}
\hline TIPO DE ECLAMPSIA & Anteparto & Intraparto & & Mort./Globa \\
\hline Total de Casos & 21 & 20 & & \\
\hline Feto vivo al ingreso & 19 & 29 & & \\
\hline Feto muerto al ingreso & 2 & 0 & & \\
\hline Repitieron convulsiones & 16 & 16 & & \\
\hline No repitieron convulsiones & 5 & 4 & & \\
\hline PARIDAD Primíparas & 16 & 16 & & \\
\hline Secundíparas & 2 & 3 & & \\
\hline Tercíparas y más & 3 & 1 & & \\
\hline PARTOS Espontáneos & 11 & 8 & & \\
\hline Inducidos & 1 & 0 & & \\
\hline Fórceps & 5 & 10 & & \\
\hline Cesáreas & 3 & 2 & & \\
\hline Sin parto & 1 & 0 & & \\
\hline Peso Fetal 1.000 a 1.500 grs. & 2 & 1 & & \\
\hline 1.501 a 2.000 & 8 & 2 & & \\
\hline 2.001 a 2.500 & 3 & 11 & & \\
\hline 2.501 a 3.000 & 2 & 2 & & \\
\hline 3.001 a 3.500 & 3 & 3 & & \\
\hline 3.501 a $4.000 "$ & 2 & 1 & & \\
\hline Feto al nacer VIVOS & 13 & 15 & & \\
\hline MUERTOS & 7 & 5 & 12 & $=29.26 \%$ \\
\hline Mortalidad Perinatal & 7 & 3 & 10 & $=24.39 \%$ \\
\hline Mortalidad Fetal Global & 14 & 22 & 22 & $=53.65 \%$ \\
\hline Mortalidad Materna & 2 & 4 & 6 & $=14.63 \%$ \\
\hline
\end{tabular}




\section{OBSERVACIONES}

Al analizar este primer grupo de tratamiento encontramos: 1) de 98 casos, 43 pacientes repitieron convulsiones lo cual dá un $43.89 \%$ de fracaso inicial desde el punto de vista terapéutico. 2) respecto al tipo de parto podemos observar que 31 casos fueron intervenidos con cesárea, o sea, un $31.63 \%$ índice bastante alto si se tiene en cuenta que la intervención quirúrgica se practicó con la exclusiva indicación de la eclampsia sin que mediara justificación obstétrica diferente. 3 ) la mortalidad materna global fué de $18.36 \%$ correspondiente a 18 pacientes. 4) la mortalidad fetal global fue de $34.04 \%$ pues 32 fetos fallecieron.

\section{SEGUNDO GRUPO}

Las causas de esta mortalidad materna de $14.63 \%$ se detallan a continuación en las síntesis de las historias clínicas:
Rev. Col. Obst. y Ginec.

\section{ECLAMPSIA ANTEPARTO: 2}

1) Historia $\mathbf{N}^{9}$ 23.905, paciente que presenta 4 ataques antes del tratamiento $y$ numerosos después. Coma y defunción.

2) Historia $\mathbf{N}^{0} \mathbf{3 5 . 1 9 7}$, paciente que presenta 2 ataques antes del tratamiento. Coma y defunción.

\section{ECLAMPSIA INTRAPARTO: 4}

1) Historia No 24.884, paciente que presenta 9 ataques antes del tratamiento y 2 después. Coma y defunción.

2) Historia $\mathbf{N}^{\mathbf{0}} \mathbf{2 3 . 9 4 9}$, paciente que presentó 2 ataques antes del tratamiento y 2 después. Ocho días después fallece por edema pulmonar.

3) Historia $\mathbf{N}^{0}$ 23.099, paciente que presentó 1 ataque antes del tratamiento y numerosos después. Muere a las 36 horas de su ingreso.

4) Historia No $\mathbf{3 3 . 2 8 5}$, paciente que fallece por coma urémico y glomérulonefritis supurada (datos de autopsia).

TERCER GRUPO

\begin{tabular}{|c|c|c|c|c|}
\hline TIPO DE ECLAMPSIA & Anteparto & Intraparto & Postparto & Mort./Global \\
\hline Total de Casos & 22 & 39 & 6 & \\
\hline Feto vivo al ingreso & 17 & 35 & 0 & \\
\hline Feto muerto al ingreso & 5 & 4 & & \\
\hline Repitieron convulsiones & 11 & 23 & 3 & \\
\hline No repitieron convulsiones & 11 & 16 & 3 & \\
\hline PARIDAD Primíparas & 14 & 29 & 6 & \\
\hline Secundíparas & 3 & 5 & 0 & \\
\hline Tercíparas y más & 5 & 5 & 0 & \\
\hline PARTOS Espontáneos & 10 & 14 & & \\
\hline Fórceps & 1 & 18 & & \\
\hline Cesáreas & 8 & 4 & & \\
\hline Ventosas & 0 & 2 & & \\
\hline Peso Fetal 1.000 a 1.500 grs. & 5 & 4 & & \\
\hline 1.501 a 2.000 & 4 & 5 & & \\
\hline 2.001 a 2.500 & 4 & 7 & & \\
\hline 2.501 a $3.000 "$ & 3 & 17 & & \\
\hline 3.001 a $3.500 "$ & 2 & 4 & & \\
\hline Feto al nacer VIVOS & 11 & 31 & & \\
\hline MUERTOS & 11 & 8 & & $19=31.14 \%$ \\
\hline Mortalidad Perinatal & 5 & 2 & & $7=10.4 .1 \%$ \\
\hline Mortalidad Fetal Global & 16 & 10 & & $26=42.62 \%$ \\
\hline Mortalidad Materna & 4 & 3 & 0 & $7=10.44 \%$ \\
\hline
\end{tabular}




\section{OBSERVACIONES}

El análisis de este segundo grupo nos demuestra: 1) 32 pacientes repitieron las convulsiones lo cual equivale al $78.04 \%$ de fracaso en el tratamiento inicial. 2) Se intervino mediante cesárea al $12.19 \%$ de las pacientes, índice alto si se tiene en cuenta que la indicación de la operación fué solamente la toxemia. 3) La mortalidad materna fué de $14.63 \%$ correspondiente a 6 pacientes fallecidas.

\section{TERCER GRUPO}

Obsérvense los datos de mortalidad fetal y materna (42.62 y $10.44 \%$ respectivamente). Las causas de las defunciones maternas se resumen en las siguientes historias clínicas:

\section{ECLAMPSIA ANTEPARTO: 4}

1) Historia $N^{0} \mathbf{3 1 . 8 2 4}$, paciente que presentó un ataque y entró en coma. Fallece 47 horas después de su ingreso por accidente cerebrovascular.
2) Historia № 54.903, paciente que presentó 4 ataques antes del tratamiento, entra en estado de coma y muere a las 23 horas de haber ingresado. Autopsia: Hemorragia intrapeduncular del puente y edema pulmonar.

3) Historia $\mathrm{N}^{0}$ 57.650, paciente que presenta 4 ataques antes del tratamiento y 2 después. Fallece. Punción lumbar: (líquido hemático) (¿Hemorragia cerebral?).

4) Historia $\mathrm{N}^{9} \mathbf{5 6 . 1 2 8 ,}$, paciente que ingresa en coma, fallece y se le practica cesárea Postmortem. Autopsia: Hemorragia cerebral extensa.

\section{ECLAMPSIA INTRAPARTO: 3}

1) Historia $\mathbf{N}^{0}$ 28.597, paciente que presentó 1 ataque antes del tratamiento y 2 después.

2) Historia $\mathbf{N}^{0} \mathbf{3 5 . 4 6 8}$, paciente que presentó 6 ataques antes del tratamiento. Muere 6 horas después de su ingreso.

3) Historia $\mathbf{N}^{o} \mathbf{5 2 . 1 1 3}$, paciente que presentó 1 ataque antes del tratamiento y 3 después. Muere 11 días después. Autopsia: Eclampsia "Grave".

\section{CUARTO GRUPO}

\begin{tabular}{|c|c|c|c|c|c|}
\hline TIPO DE ECLAMPSIA & Anteparto & Intraparto & Postpario & & Mort./Global \\
\hline Total de Casos & 180 & 121 & 45 & & \\
\hline Feto vivo al ingreso & 156 & 106 & & & \\
\hline Feto muerto al ingreso & 24 & 15 & & & \\
\hline Repitieron convulsiones & 26 & 32 & 6 & & \\
\hline No repitieron convulsiones & 154 & 89 & 39 & & \\
\hline PARIDAD Primíparas & 126 & 88 & 20 & & \\
\hline Secundíparas & 14 & 11 & 13 & & \\
\hline Terciparas & 40 & 19 & 12 & & \\
\hline PARTOS Espontáneos & 108 & 54 & & & \\
\hline Inducidos & 21 & 0 & & & \\
\hline Fórceps & 36 & 51 & & & \\
\hline Cesáreas & 13 & 15 & & & \\
\hline Espátulas & 1 & 1 & & & \\
\hline Ventosas & 1 & 0 & & & \\
\hline Peso Fetal 1.000 a 1.500 grs. & 33 & 8 & & & \\
\hline 1.501 a $2.000 "$ & 34 & 9 & & & \\
\hline 2.001 a 2.500 & 35 & 22 & & & \\
\hline 2.501 a 3.000 & 30 & 38 & & & \\
\hline 3.001 a 3.500 & 12 & 26 & & & \\
\hline 3.501 a $4.000 "$ " & 3 & 8 & & & \\
\hline Fetos al nacer VIVOS & 122 & 101 & & & \\
\hline MUERTOS & 55 & 20 & & 75 & $=24.91 \%$ \\
\hline Mortalidad Perinatal & 15 & 12 & & 27 & $=8.97 \%$ \\
\hline Mortalidad Fetal Global & 70 & 32 & & 102 & $=33.88 \%$ \\
\hline Mortalidad Materna & 9 & 4 & 4 & 17 & $=4.91 \%$ \\
\hline
\end{tabular}




\section{OBSERVACIONES}

Al analizar este grupo encontramos que: 1) 37 pacientes repitieron convulsiones lo cual corresponde a un $55.22 \%$ de fracaso inicial del tratamiento. 2) Se practicó la intervención cesárea a 12 pacientes lo cual equivale a $19.67 \%$ de frecuencia para este tipo de operación determinada solamente por el fracaso en el tratamiento médico de la eclampsia. 3) La mortalidad fetal global fué de 26 que corresponden a un $42.62 \%$. 4) La mortalidad materna global fué de 7 pacientes a las cuales corresponde un porcentaje de $10.44 \%$.

\section{CUARTO GRUPO}

Queremos hacer hincapié en el porcentaje de las mortalidades materna y fetal sobre cuyas cifras insistiremos adelante. Veremos en detalle las circunstancias y las causas de las defunciones maternas en los siguientes resúmenes de las historias clínicas correspondientes:

\section{ECLAMPSIA ANTEPARTO: 9}

1) Historia $\mathbf{N}^{0} \mathbf{5 8 . 4 5 2}$, paciente que ingresó en estado de coma. Permaneció en el Instituto 55 minutos. Se practicó cesárea postmortem.

2) Historia $N^{0}$ 68.457, paciente de 23 años que ingresó en estado de coma habiendo padecido 40 minutos antes un ataque. Fallece a los 50 minutos de su ingreso. Autopsia: Hemorragias múltiples en cerebro, hígado, corazón y diafragma.

3) Historia $\mathbf{N}^{0}$ 68.729, paciente que presenta 15 ataques antes de su ingreso. No repitió convulsiones en el Servicio. Muere a los 7 días de su ingreso en insuficiencia renal aguda. Autopsia: Hemorragia subaracnoidea, enfermedad vascular crónica hipertensiva.

4) Historia № $\mathbf{3 8 . 8 3 1}$, paciente que presentó 4 ataques antes de su ingreso. No repitió las convulsiones. Presentó en el puerperio accidente cerebrovascular. Autopsia: Hematoma intracerebral. Meningitis TBC.
5) Historia No 72.894, paciente que ingresó después de haber presentado 9 ataques no repitió las convulsiones pero permaneció en coma durante 8 horas y falleció por accidente cerebrovascular.

6) Historia $\mathbf{N}^{\mathbf{9}} \mathbf{7 4 . 3 0 1}$, paciente que ingresó en estado de coma por accidente cerebrovascular declarado luego de un ataque. Autopsia: Hemorragia subaracnoidea e intracerebral. Hipertensión arterial. Muere a las 6 horas.

7) Historia. Paciente que ingresa en estado de coma por accidente cerebrovascular. Se ignora la evolución previa. En el Servicio no hizo convulsiones.

8) Historia $\mathbf{N}^{0} \mathbf{2 7 . 0 1 2}$, paciente que presentó 4 ataques antes del ingreso. No repitió las convulsiones. Falleció a las 9 horas por accident cerebrovascular. Cesárea postmortem.

9) Historia $\mathbf{N}^{0} \mathbf{9 6 . 1 0 5}$, paciente que presentó 1 ataque antes del ingreso y que fallece 7 horas después de hemorragia cerebral severa comprobada a la autopsia. Se practicó cesárea post mortem.

\section{ECLAMPSIA INTRAPARTO: 4}

1) Historia No 61.270, paciente de 22 años que ingresó en estado comatoso con signos de hemorragia cerebral y desprendimiento prematuro de placenta normoinserta. Murió a los 60 minutos de su ingreso.

2) Historia $\mathbf{N}^{\mathbf{0}} \mathbf{7 6 . 4 5 4}$, paciente que presenta 8 ataques antes del ingreso y 2 después. Parto gemelar con fetos muertos. Muere por necrosis tubular aguda. Autopsia: Hemorragias subpiales.

3) Historia $\mathbf{N}^{0} \mathbf{3 0 . 6 2 6}$, paciente que hizo 7 ataques antes de su ingreso y 2 después del tratamiento, en el último hizo accidente cerebrovascular que provocó la muerte. Tratamiento I.M.I. insuficiente.

4) Historia $\mathbf{N}^{0} \mathbf{7 0 . 1 3 2}$, paciente que presentó 2 ataques antes del ingreso y 5 después. Falleció 2 días después del parto. Autopsia: Bronconeumonía bilateral. Edema agudo del pulmón. Hemorragia cerebral en los núcleos grises izquierdos. 


\section{ECLAMPSIA POSTPARTO: 4}

1) Historia $\mathbf{N}^{0}$ 53.676, paciente que ingresa después de numerosos ataques y parto en la casa. Coma y muerte. Autopsia: Hemorragia cerebral - Edema pulmonar.

2) Hisioria $\mathbf{N}^{0} \mathbf{7 6 . 2 4 2}$, paciente que ingresa después de haber tenido su parto. Hace un ataque y accidente cerebrovascular. Fallece a las 11 horas de su ingreso.

3) Historia № 76.025, paciente que presenta muerte súbita. Autopsia: Insuficiencia renal aguda ( $\sin$ diagnóstico clínico).

4) Historia No 91.242, paciente que presenta 20 ataques antes del tratamiento -ingresopresenta accidente cerebrovascular. Permaneció 3 horas hospitalizada. Autopsia: Hemorragia cerebral, inundación ventricular.

\section{OBSERVACIONES}

Debemos insistir en las siguientes deducciones: 1) De 346 pacientes tratadas por el método " $\mid \mathrm{MI}$ " sola- mente 64 de ellas o sea el $18.49 \%$ repitieron las convulsiones una vez iniciado el tratamiento. Cabe anotar que en algunas de ellas el fracaso inicial se debió a la insuficiencia en la administración de la terapia. 2) Solamente se practicaron 28 cesáreas $(9.30 \%)$ que tuvieron como indicación alguna causa obstétrica pura ya que no se efectuó ninguna de ellas por la Eclampsia. 3) En el $28.90 \%$ de los casos se aplicó el fórceps como medida profiláctica. 4) La mortalidad fetal global fué de $33.88 \%$. 5) La mortalidad materna global fué solamente de $4.91 \%$.

\section{Análisis de la Mortalidad}

Hemos querido hacer de este aspecto del trabajo un comentario general al respecto. A fin de observar comparativamente las cifras presentamos el cuadro siguiente que incluye los datos de los distintos grupos de tratamiento:

\begin{tabular}{lccrr} 
GRUPO & I & II & III & IV \\
\hline Mortalidad Fetal & $34.04 \%$ & $53.65 \%$ & $42.62 \%$ & $33.88 \%$ \\
Mortalidad Materna & $18.36 \%$ & $14.63 \%$ & $10.44 \%$ & $4.91 \%$
\end{tabular}

Deseamos hacer énfasis en el hecho de que estas estadísticas no han sido depuradas. Deliberadamente prescindimos de la depuración a fin de presentar hechos escuetos e imparciales. No hemos querido mostrar solamente la bondad de un determinado tipo de tratamiento sino también observar la trascendencia global desde el punto de vista pronóstico de la Eclampsia. Para confirmar lo anterior basta con observar cómo la mortalidad fetal con el tratamiento "IMI" (33.$88 \%$ ) se hubiera reducido a $20.93 \%$ solamente con excluir de la estadística aquellos casos de pacientes que ingresaron al Instituto portando un feto muerto in útero. De igual manera se hubiera podido reducir aún más la mortalidad fetal dejando a un lado aquellos casos con fetos cuyo peso lindaba con la viabilidad o aún estaban por debajo de ella.

Procedimos de igual manera con la mortalidad materna de cuyas estadísticas hubiéramos podido excluir aquellos casos cuya gravedad intrínseca por las complicaciones a que hubieran dado lugar determinaban la muerte fuera cual fuera el tratamiento empleado y bajo cuyo pronóstico fatal ingresaron al Servicio. 
A pesar de las circunstancias anteriores veremos en el cuadro siguiente cómo la mortalidad materna por
Eclampsia ha descendido de manera radical a través de los últimos 8 años en el Instituto Materno Infantil.

\begin{tabular}{rrrrrcr}
\hline Año & $\begin{array}{c}\text { Total de } \\
\text { Partos }\end{array}$ & $\begin{array}{c}\text { Número de } \\
\text { Eclampsias }\end{array}$ & $\begin{array}{c}\text { Incidencia de } \\
\text { Eclampsias } \%\end{array}$ & $\begin{array}{c}\text { Pacientes muertas } \\
\text { por Eclampsia }\end{array}$ & $\begin{array}{c}\text { Porcentaje } \\
\text { de } \\
\text { portalidad } \\
\text { Eclampsia }\end{array}$ \\
\hline 1954 & 7.004 & 55 & $0.78 \%$ & 10 & $0.14 \%$ \\
1955 & 7.526 & 46 & $0.61 \%$ & 10 & $0.13 \%$ \\
1956 & 8.738 & 53 & $0.60 \%$ & 7 & $0.08 \%$ \\
1958 & 9.281 & 54 & $0.58 \%$ & 5 & $0.05 \%$ \\
1959 & 10.648 & 84 & $0.78 \%$ & 2 & $0.02 \%$ \\
1960 & 11.916 & 86 & $0.74 \%$ & 8 & $0.06 \%$ \\
1961 & 13.165 & 102 & $0.77 \%$ & 3 & $0.02 \%$ \\
1962 & 13.911 & 70 & $0.50 \%$ & 3 & $0.02 \%$ \\
\hline
\end{tabular}

Obsérvese cómo en el año 1954 la mortalidad por Eclampsia era para un volumen de 7.004 pacientes de $0.14 \%$ mientras que en el año 1962 para 13.911 solamente fué de $0.02 \%$.

El siguiente cuadro estadístico es- obtenidos en trabajos tanto nacionatablece la comparación de resultados les como extranjeros:

\begin{tabular}{|c|c|c|c|c|c|}
\hline AUTOR & PAIS & Mortalidad & Materna & Mortalid & Fetal \\
\hline Ortiz y Gamboa y cols. (4) & Colombia & $22.320 .57 y$ & $11.84 \%\left({ }^{*}\right)$ & & 44 \\
\hline Dieckman (5) & U. S. A. & $21.0013 .00 y$ & $16.00 \%(* *)$ & & 22 \\
\hline Aguero y cols. (6) & Venezuela & 20.4 & $\%$ & & $23 \%$ \\
\hline Olivares y cols. (7) & México & 20.0 & $\%$ & & $45.90 \%$ \\
\hline Sanz Aráoz (8) & Colombia & 12.5 & $\%$ & & $25 \%$ \\
\hline Silva y cols. (9) & Colombia & 12.0 & $\%$ & & 39 \\
\hline García y Bunster (10) & Chile & 10.69 & $\%$ & & \\
\hline Arcay Sola y cols. (11) & Venezuela & 8.45 & $\%$ & & $30.70 \%$ \\
\hline Mayorga y cols. (12) & Chile & 6.9 y 10.2 & $2 \% \quad(* *)$ & $18.1 \mathrm{y}$ & $35.7 \%$ \\
\hline Adams y cols. (13) & U. S. A. & 6.8 & $\%$ & & $23.5 \%$ \\
\hline Gómez Palacino (14) & Colombia & 6.2 & $\%$ & & $43.1 \%$ \\
\hline PUGA y cols. (15) & Chile & 5.0 & $\%$ & & $33.4 \%$ \\
\hline Páez Vargas & Colombia & 4.91 & $\%$ & & $33.88 \%$ \\
\hline Martínez y cols. (16) & Colombia & 4.0 & $\%$ & & $12.9 \%$ \\
\hline
\end{tabular}

(*) Mortalidad preparto, intraparto y postparto.

(**) Con cesárea y con tratamiento médico.

Del estudio del cuadro estadístico anterior se concluye que los datos aportados en los trabajos nacionales son ampliamente satisfactorios puesto que demuestran dos hechos fundamentales: 1) Que la mortalidad tanto materna como fetal se ha ido reduciendo en forma progresiva y 2 )
Que de las estadísticas consultadas dichos datos son los más reducidos. Las estadísticas publicadas por Martínez, Gómez, Sánchez y Silva, (16) cuyos resultados fueron obtenidos también con el tratamiento " $|M|^{\prime \prime}$ al igual que las nuestras, son francamente alentadoras. 


\section{RESUMEN}

Se hace un recuento de la evolución histórica de la terapia anticonvulsivante empleada en la Eclampsia a partir de 1745. Se esquematizan los diversos tipos de tratamiento empleados en el INSTITUTO MATERNO INFANTIL DE BOGOTA a partir del año de 1954. Se presentan los resultados obtenidos con dichos tratamientos. Se establecen las comparaciones estadísticas respectivas así como también con los datos obtenidos en publicaciones extranjeras.

\section{CONCLUSIONES}

1. El tratamiento de la Eclampsia a través de los tiempos ha tropezado con los inconvenientes inherentes al desconocimiento de la razón íntima y fundamental del síndrome.

2. Las modernas adquisiciones y aportes de la medicina han permitido vislumbrar y aclarar algunos de los fenómenos, signos y síntomas de la toxemia gravídica.

3. El conocimiento de la fisiopatología de la Eclampsia ha permitido la modificación del criterio terapéutico haciendo racional y lógico su tratamiento.

4. El descubrimiento de medicamentos tales como los neuroplégicos ha hecho menos sombrío el pronóstico de la Eclampsia.

5. La fundación y organización de Departamentos o Servicios especializados dentro de las Instituciones asistenciales ha dado magníficos resultados.

6. La dedicación de personal médico a la investigación ha permitido el avance, conocimiento y divulgación de procedimientos cada vez más efi- caces en el control y tratamiento de la toxemia del embarazo.

7. La mortalidad materna ha descendido, de acuerdo con nuestra revisión de 18.36 a $4.91 \%$ en el corto lapso de 8 años.

8. La mortalidad fetal, no ha disminuido en la misma proporción pero los resultados a pesar de ello son satisfactorios.

9. En nuestro medio los resultados obtenidos con el tratamiento "l. M.I." son muy halagadores y demuestran la bondad elocuente de sus bases fisiopatológicas y método.

10. Del estudio comparativo hecho en este trabajo podemos concluir que cl tratamiento "IMI" es el que mejores resultados ha dado en nuestro medio y por lo tanto es recomendable su uso.

11. La adopción de medidas profilácticas y una campaña educacional y sanitaria se imponen por parte de los organismos respectivos pues la incidencia de toxemia en nuestro país refleja graves fallas en el sistema.

\section{BIBLIOGRAFIA}

1 WALKER, W. and BAKER, Jr. A comparison study of antihypertensive drug therapy and the modified Stroganoff metod in the management of severe toxemia of pregnancy. Am. J. Obst. y Gynec. 81: 16, 1961.

2 GOMEZ PALACINO, J. A. Etiopatogenia de la Toxemia Gravídica. Rev. Colomb. de Obs. y Gin. 10: 399, 1959.

3 SANCHEZ TORRES, F. Tratamiento de la Preeclampsia-Eclampsia. Rev. Colomb. de Obs. y Gin. 10: 410, 1959.

4 ORTIZ y cols. Mortalidad Materna. Rev. Colomb. de Obs. y Gin. 5: 951954.

5 DIECKMAN, W. J.: The Toxemias of Pregnancy, ed. 2. St. Louis, 1954, The C. V. Mosby Company. 
6 AgUeRO, O. y cols. Toxemias en la Maternidad. "Concepción Palacios" en 1954 Rev. de Obs. y Gin. de Venezuela. 4: 1.285, 1955.

7 OLIVARES, J. y cols. Avances en estudio y tratamiento de la Toxemia Gravídica. Rev. de Obs. y Gin. de México. 16: 479, 1961.

8 SANZ, MARIO. Tratamiento de la Eclampsia del Puerperio con Pentotal. Rev. Colomb. de Obs. y Gin. 8: 135, 1957.

9 SILVA y cols. Tratamiento de la Toxemia Gravídica. Rev. Colomb. de Obs. y Gin. 9: 261, 1958.

10 GARCIA y BUNSTER. Mortalidad Materna. Rev. Chilena de Obs. y Gin. 26: 472, 1961.

11 ARCAY SOLA y cols. Toxemias del Embarazo. Rev. de Obs. y Gin. de Venezuela. 19: $313,1959$.
12 MAYORGA, L. y cols. Cesárea en la Eclampsia. Rev. Chilena de Obs. y Gin. 26: $294,1961$.

13 ADAMS, J. Q. and C. Meron. W. The management of Eclampsia. Am. J. Obst. y Gynec. 80: 253, 1960.

14 GOMEZ PALACINO, J. A. Sulfato de Magnesia Intrarraquídeo en el tratamiento de la Eclampsia. Rev. Colomb. de Obs. y Gin. 8: $141,1957$.

15 PUGA, J. A. y cols. El tratamiento de la Eclampsia convulsiva con perfusión prolongada de Pentotal Sódico. Revista Chilena de Obs. y Gin. 26: 434, 1961.

16 MARTINEZ C. y cols. Resultados en 125 casos de Eclampsia. Memorias del III Congreso Uruguayo de Ginecotocología. Tomo II: 193.1960.

\title{
PRIMER CURSO DE CELIOSCOPIA EN GINECOLOGIA SEGUNDO CURSO DE COLPOSCOPIA Y CITOLOGIA EN GINECOLOGIA
}

\author{
del 12 al 17 de Diciembre de 1966
}

BARCELONA

Sə llevará a cabo en el Instituto Provincial de Maternidad de Barcelona y en el Centro de Lucha contra el Cáncer de la misma ciudad.

\section{Inscripciones:}

Señorita M. TRIAS - C. L. C.

Instituto Provincial de Maternidad de Barcelona

Las Cortes - Barcelona

Valor del Curso: 3.000 ptas. 